\title{
Image-Based, Fiber Guiding Scaffolds: A Platform for Regenerating Tissue Interfaces
}

\author{
Chan Ho Park, PhD, ${ }^{1,2,{ }^{*}}$ Hector F. Rios, DDS, PhD, ${ }^{1}$ Andrei D. Taut, BS, ${ }^{1}$ Miguel Padial-Molina, DDS, PhD, \\ Colleen L. Flanagan, MS, ${ }^{2}$ Sophia P. Pilipchuk, BS, ${ }^{2}$ Scott J. Hollister, PhD, ${ }^{2-4}$ \\ and William V. Giannobile, DDS, DMedSc ${ }^{1,2}$
}

In the oral and craniofacial complex, tooth loss is the most commonly acquired disfiguring injury. Among the most formidable challenges of reconstructing tooth-supporting osseous defects in the oral cavity is the regeneration of functional multi-tissue complexes involving bone, ligament, and tooth cementum. Furthermore, periodontal multi-tissue engineering with spatiotemporal orientation of the periodontal ligament (PDL) remains the most challenging obstacle for restoration of physiological loading and homeostasis. We report on the ability of a hybrid computer-designed scaffold-developed utilizing computed tomography-to predictably facilitate the regeneration and integration of dental supporting tissues. Here, we provide the protocol for rapid prototyping, manufacture, surgical implantation, and evaluation of dual-architecture scaffolds for controlling fiber orientation and facilitating morphogenesis of bone-ligament complexes. In contrast to conventional singlesystem methods of fibrous tissue formation, our protocol supports rigorous control of multi-compartmental scaffold architecture using computational scaffold design and manufacturing by 3D printing, as well as the evaluation of newly regenerated tissue physiology for clinical implementation.

\section{Introduction}

$\mathbf{P}$ ARADIGMS OF TISSUE engineering and regenerative medicine have led to a dramatic shift from prosthetic tissue replacement toward tissue regeneration using biomaterials combined with biologics. ${ }^{1,2}$ Although regenerative medicine holds promise as a prominent future therapeutic strategy, only a limited number of studies have achieved success in multipletissue regeneration and functional interfacial tissue integration, as evident in ligament-bone or cartilage-bone complexes. ${ }^{3-6}$ To overcome these therapeutic difficulties, multi-phasic scaffolds or physical gradient constructs have been designed and used for multiple tissue formation within a single system ${ }^{7,8}$ as well as for bioactive molecule-induced tissue morphogenesis and regeneration. ${ }^{9}$ Various in vitro approaches highlight the importance of geometric control on tissue regeneration/ morphogenesis using a variety of cell types, ${ }^{10-12}$ but geometric influences on spatiotemporal tissue regeneration and functional healing have had limited assessment in vivo. 3,13

Dental-supportive connective tissue, known as periodontal ligament (PDL), is anchored between mineralized tissue surfaces and plays a key role in the transmission of biomechanical proprioception, $3,13,14$ adaptive responses of masticatory cyclic movements, ${ }^{13}$ shock absorption of external forces, and tooth movement. These critical functions are related to the orientation of collagenous fiber bundles found in the PDL interface. In addition, ${ }^{15}$ the PDL interface is composed of a heterogeneous cell population containing stem cell-like multipotent cells. ${ }^{16-18}$ For the regeneration of functional periodontal complexes, the orientation of fibrous connective PDL tissues should exhibit perpendicular or oblique angulation to the tooth root surface. However, lack of control over the ligamentous fiber orientation remains a limitation for the regeneration of fibrous-mineralized tissue complexes.

This protocol provides an interdisciplinary and translational guide for the regeneration of the fibrous tissuemineralized tissue complex within a single engineered environment. We expect this strategy for the functional restoration of the periodontal complex to provide insights into other interdisciplinary approaches for tissue interface regeneration, with eventual translation to the clinical arena.

\section{Experimental comparison with the conventional method}

In addition to the strategies used by Oshima et al., ${ }^{19}$ various other combinations of therapeutic gene delivery, ${ }^{20-22}$ bioactive molecule therapy, ${ }^{23,24}$ and scaffolding material integration ${ }^{25-28}$

\footnotetext{
${ }^{1}$ Department of Periodontics and Oral Medicine, School of Dentistry; ${ }^{2}$ Department of Biomedical Engineering, College of Engineering; ${ }^{3}$ Department of Surgery, School of Medicine; ${ }^{4}$ Department of Mechanical Engineering, College of Engineering, University of Michigan, Ann Arbor, Michigan.

${ }^{*}$ Current affiliation: Department of Periodontology, School of Dentistry, Seoul National University, Seoul, South Korea.
} 
have been developed and evaluated for multi-tissue regeneration. ${ }^{29-33}$ Despite the outlined efforts to orchestrate multitissue formation in vivo, lack of control over the fibrous connective tissue orientation and the integration between ligament and mineralized tissues limit the preclinical and clinical application of multi-tissue regenerative therapies. Conventional scaffold design and manufacturing methods do not allow for compartmentalization of differing tissues and, thus, subsequent re-organization and integration of fibrous and mineralized tissues. To address these limitations, we utilized computational scaffold design and 3D printing of oriented structures to provide compartmentalized biologic delivery in an in vivo rodent fenestration model. In periodontal regeneration studies, the rodent fenestration model has often been used to evaluate regenerative strategies and outcomes under biomechanical loading conditions. ${ }^{34,35}$

\section{Experimental design}

The customized topography and structure-generated using micro-computed tomography (micro-CT; eXplore Locus SP, GE Healthcare, Inc.) and image-based CAD/CAM system software- of the hybrid scaffold structure in our recent investigations ensures that ${ }^{1}$ the micro-architecture, shape, and adaptation of the scaffold are customized for the created defect ${ }^{2}$-the fiber-guiding portion of the scaffold controls the orientation of the Sharpey's fiber-like fibrous bundles by serving as a platform for cellular/tissue activity, and $^{3}$ the customized topography facilitates fibrous tissue integration into the mineralized layer of the tooth root surface.

\section{Development of protocol}

We previously compared the geometric control abilities of anatomically defect-fit, fiber-guiding scaffolds versus saltleached, amorphous scaffolds ${ }^{36}$ to facilitate tissue formation in an in vivo periodontal defect model. ${ }^{36,37}$ The data demonstrated that regenerated PDL had angulations similar to healthy, mature ligamentous tissues. In addition, each tissue demonstrated restored physiological function for tooth movement and alveolar bone maintenance under biomechanical masticatory loading conditions. ${ }^{37}$ Perpendicular and oblique orientation of the fiber-guiding PDL architectures to the tooth root surface promoted the integration of collagenfibrous (Sharpey's fiber) bundles into both the cementum and alveolar bone. Contrastingly, easily fabricable porogenleached amorphous scaffolds with random pore architecture led to the formation of disorganized PDL structures and unpredictable mineralized tissue infiltration. ${ }^{36}$

\section{Application of the method}

The fundamental design and fabrication methodology of biomimetic, hybrid fiber-guiding scaffolds supporting the organization and integration of periodontal interfaces is a platform technology that can be further translated to many relevant in vivo models and clinical needs for multi-tissue regeneration.

\section{Materials and Methods}

\section{Defect site three-dimensional digital image dataset and scaffold design (Fig. 1)}

A standardized osseous fenestration defect $(3 \times 2 \mathrm{~mm})$ was surgically created as previously described. ${ }^{36,38}$ Briefly, the defect was created on the buccal side of the mandible (Fig. 2a), and the cementum layer was removed from the root surface to expose the distal root of M1. Standardization of the defect was performed using a periodontal probe to verify the $3 \mathrm{~mm}$ width $\times 2 \mathrm{~mm}$ height. All animal surgeries were performed under a protocol approved by the University of Michigan-University Committee on Use and Care of Animals. After defect creation, rats were euthanized and mandibles were collected for micro-CT scanning. Mandibulae were scanned at a resolution of $18 \mu \mathrm{m}$. The scanner was set up with $2 \times 2$ binning sizes, $80 \mu \mathrm{A}$ beam current, $80 \mathrm{kV}$ accelerated potential voltage, and 0.02 -inch aluminum (Al) filter in order to obtain image datasets. Ex vivo micro-CT was used for rendering three-dimensional (3-D) images of the surgically created defects (Fig. 2a). Ex vivo micro-CT scanning is preferred, because (1) in vivo micro-CT scanning of live animals does not provide an accurate depiction of PDL interface topology $(150-200 \mu \mathrm{m})$, and (2) natural animal movement can lead to artifacts. ${ }^{39}$ To design the custom-fit scaffold for each anatomical defect, a 3-D-generated digital image dataset of the periodontal defect site was first converted to the surface geometry, stereolithography file format (.STL) using MicroView Analysis +2.1.2 (GE Healthcare, Inc.). The STL-formatted file was opened using Unigraphics NX 5 (Siemens PLM software). After capturing the periodontal defect image dataset, the exterior borders of the bone region and PDL interface were created using periodontal defect topography in the STL surface image. ${ }^{36}$

For design of PDL interface architecture, the supporting structure with $0.250 \mathrm{~mm}$ width was designed and three $0.225 \mathrm{~mm}$-diameter cylindrical architectures per layer were oriented perpendicularly to the root surface (Fig. 2b). ${ }^{36}$ A total of four layers were created with a $0.175 \mathrm{~mm}$ gap distance in between. The cell-loading capacity of the PDL

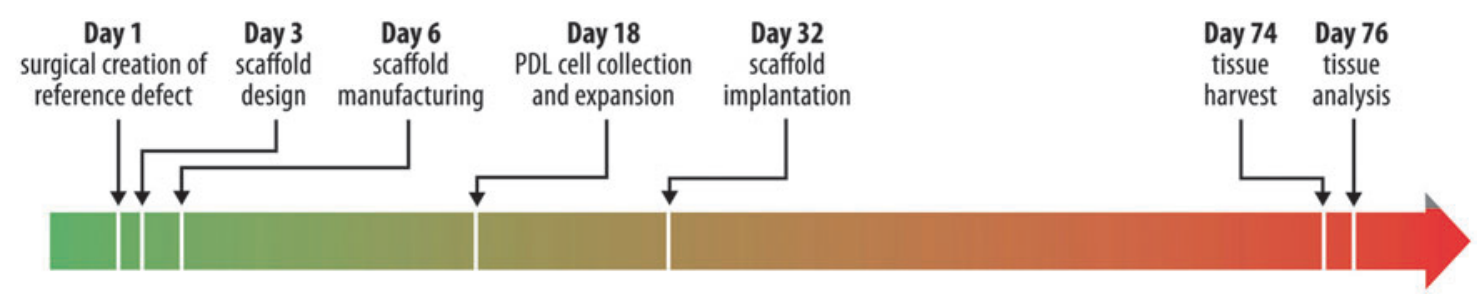

FIG. 1. Timeline for scaffold design, manufacture, implantation, and evaluation. Periodontal ligament (PDL) cell harvest and expansion lasted $\sim 14$ days, while tissue healing and regeneration was evaluated over a duration of 6 weeks. Along with additional procedures, the entire process spanned $\sim 2.5$ months. Color images available online at www.liebertpub.com/tec 

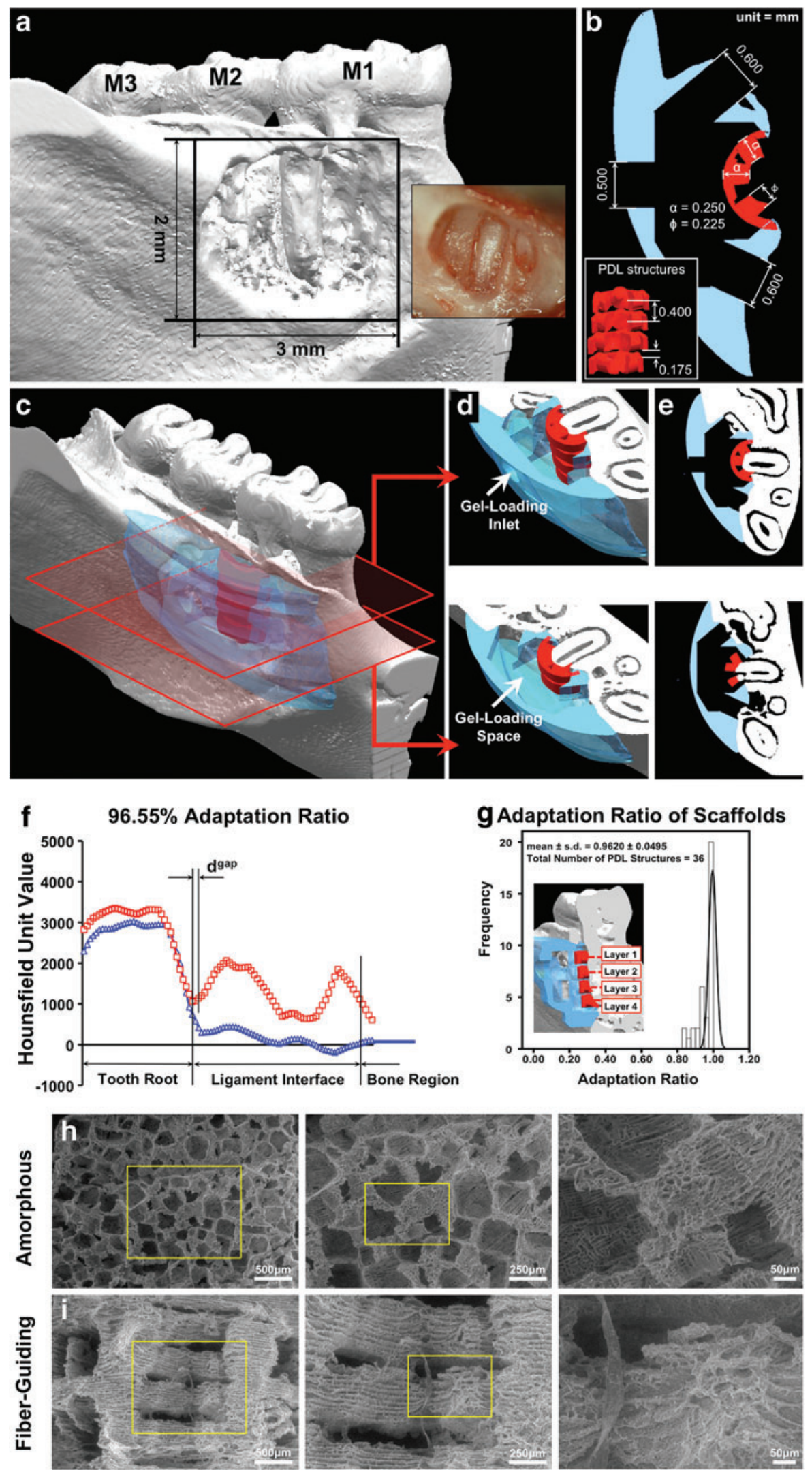

FIG. 2. Micro-computed tomographic (Micro-CT) image of mandibular osseous defect and scaffold design/dimensions. (a-e) The adaptation ratio and statistical evaluation of the scaffold reliability calculated along the entire PDL interface distance $(250 \mu \mathrm{m})$ within the osseous defect site. (f, g) Scanning electron microscopic (SEM) images showcase the contrasting internal architecture of the random-porous (micro-/nano-scale) and fiberguiding (micro-/submicron scale) scaffolds. (h, i) (Reprinted with permission from [36]). Color images available online at www.liebertpub.com/tec 
architecture space in the fiber-guiding scaffold was $2 \mu \mathrm{L} .{ }^{36}$ Magics 15 (Materialise NV) was used to improve scaffold adaptation to the osseous defect geometry. Designed PDL architectures were subtracted from STL-formatted, 3-D surface topological defect to generate an anatomically fit scaffold.

For design of the bone region architecture, a block with rounded outer borders was created based on the mandibular defect STL-image dataset. The external bone topology of the scaffold was designed to cover the entire defect (minimum dimensions of $3 \times 7 \mathrm{~mm}$ over the surgically created defect surface). The block was $<5 \mathrm{~mm}$ thick and $10 \mathrm{~mm}$ in length, because scaffolds larger than $5 \times 10 \mathrm{~mm}$ can irritate the inner muscle/skin and, eventually, become exposed. ${ }^{40}$ The $4 \mu \mathrm{L}$ cell/gel-containing spaces were designed with an opened window to allow for interactions between native tissues and loaded cells. The bone-contact surface had a total of four $0.600 \times 0.500 \mathrm{~mm}$ windows to enable tissue infiltration, bone ingrowth, and generation of tooth-supporting structures. One $0.500 \mathrm{~mm}$ hole was created for cell-suspension fibrin gel loading on the buccal side of the mandible. ${ }^{36}$

After creating PDL interface and bone region architectures, both structures were assembled into a single system. Using NX5, the gel-containing portion, four opened windows, and gel-loading inlet were assembled to make one 3-D feature from which the loading space from the designed bone topology was then subtracted. This designed feature was converted to an STL-file format in millimeters and opened in Magics 15. The final designed scaffold was topologically subtracted from the STL-generated periodontal defect feature. After the manufacturing of scaffolds, the adaptation ratio was calculated between the scaffold and defect topology to quantify the fit of the scaffold.

\section{Fiber-guiding scaffold manufacturing}

A wax mold with $13.0 \mathrm{~mm}$ diameter and $3.50 \mathrm{~mm}$ height was digitally created with four fiber-guiding structures for the rapid prototyping technique. Twenty-five finalized mold files were loaded and organized on one platform for the 3-D wax printer, ModelMaker II (Solidscape, Inc.). After arranging the molds, the file format was converted to .mm2, saved, and loaded onto the printer. The printing time was $\sim 70-72 \mathrm{~h}$ per platform (for 25 wax molds) with $0.0254 \mathrm{~mm}$ slicing thickness. An $80 \times 80 \mathrm{~mm}$ square, 0.25 -inch-thick Teflon sheet was prepared with five, 0.50-inch diameter holes straight through the processed sheet for casting with poly- $\varepsilon$-caprolactone (PCL; MW 43-50 kDa, Polysciences, Inc., cat. no. 19561-50). PCL was dissolved in acetone (SigmaAldrich $^{\circledR}$ ) at $25 \mathrm{wt} / \mathrm{v} \%$ concentration and stored in a $37^{\circ} \mathrm{C}$ water bath, with the container tightly closed to prevent acetone evaporation.

In order to secure space for material casting, the molds were placed in $70 \mathrm{v} / \mathrm{v} \%$ ethanol and agitated for 100 $120 \mathrm{~min}$ to dissolve the Protobuild (green wax material; Solidscape, Inc.). Agitation should not last longer than $120 \mathrm{~min}$, because ethanol can slowly dissolve the supporting mold structure, and Protosupport (red wax material; Solidscape, Inc.) becomes fragile. After complete removal of Protobuild material, Protosupport was dried in the fume hood for $24 \mathrm{~h}$.

For casting fiber-guiding scaffolds into molds using 25 wt/v\% PCL solution, Teflon and black Delrin sheets were assembled tightly with clips, and the PCL solution was then carefully poured into the mold. While the PCL solution was still viscous, the mold was pressed into the polymer solution to complete the casting, after which the PCL-filled mold was left in the fume hood to enable acetone to fully evaporate. BioAct ${ }^{\circledR}$ VSO (Petroferm, Inc.) solvent was used to remove Protosupport in a $50^{\circ} \mathrm{C}-53^{\circ} \mathrm{C}$ water bath for $48-72 \mathrm{~h}$. Finally, $100 \%$ ethanol was utilized to remove any remaining BioAct VSO solvent for another 1-2 days. Scaffolds were stored in $70 \%$ ethanol before implanting them in vivo.

\section{Salt-leached scaffold fabrication}

Manufacturing of salt-leached scaffolds was done in accordance with a previously published protocol. $^{36}$ Briefly, PCL was dissolved in $25 \mathrm{wt} / \mathrm{v} \%$ polymeric 1,4-dioxane solution (Fisher-Scientific), and presieved salt particles were added to obtain a pore size range of $250-425 \mu \mathrm{m}$. This mixture was poured onto a glass petri-dish to yield a $2.0 \mathrm{~mm}$ PCL thickness, and PCL blocks were cut with approximate dimensions of $3 \times 2 \times 2 \mathrm{~mm}$. Before cell seeding, scaffolds were stored in $70 \%$ ethanol for 3 days and then exposed under UV for sterilization.

\section{Evaluating scaffolds for defect adaptation}

PCL scaffolds were immersed in 1.0-1.5 mL of 35\% barium sulfate solution $\left(\mathrm{BaSO}_{4}, \mathrm{E}-\mathrm{Z}-\mathrm{Paste}\right.$; E-Z-E-M Canada, Inc.) to obtain higher-intensity gray-scale Hounsfield Unit readings than the polymeric material during microCT scanning. The $35 \% \mathrm{BaSO}_{4}$-immersed PCL scaffolds were dried in the fume hood for $24 \mathrm{~h}$. Scaffolds were then placed in the periodontal osseous defect, and micro-CT scanning was performed in phosphate-buffered saline (PBS). The gap distance (distance between PDL architecture surface and tooth root surface) and entire PDL interface (distance between root surface and PDL architecture) were measured using MicroView Analysis + 2.1.2 software (GE Healthcare) for fiber-guiding scaffolds only. Adaptation ratio was calculated as the gap distance divided by the PDL interface distance:

$$
\text { Adaptation Ratio }=\frac{\mathrm{d}^{\text {gap }} \text { (gap distance) }}{\mathrm{d}^{\text {PDL }}(\text { PDL distance })}
$$

\section{Human PDL cell culture}

Following Institutional Review Board approval, primary human PDL (hPDL) cells were collected from extracted premolars and third molars of three healthy human patients with an age range of $12-50$ years. Cells in passages $4-5$ were cultivated in Dulbecco's-modified Eagle's medium (DMEM; Life Technologies, Inc.) with 10\% fetal bovine serum (Gemini Bio-Products), antibiotics (100 units $/ \mathrm{mL}$ penicillin and $10 \mu \mathrm{g} / \mathrm{mL}$ streptomycin), and $2 \mathrm{mM}$ glutamine in $100 \mathrm{~mm}$ tissue culture dishes. The culture environment was a humidified atmosphere of $5 \% \mathrm{CO}_{2}$ in air at $37^{\circ} \mathrm{C}$.

\section{Cell suspension with fibrin gel and seeding of scaffolds}

Bovine plasma fibrinogen (Sigma-Aldrich) was dissolved in DMEM at $5 \mathrm{mg} / \mathrm{mL}$ and sterilized with a $0.2 \mu \mathrm{m}$ syringe filter (Nalgene ${ }^{\circledR}$ ). Bovine plasma thrombin (Sigma-Aldrich) was dissolved in Hanks' Balanced Salt Solution (HBSS; Invitrogen Corporation) at $100 \mathrm{U} / \mathrm{mL}$. Approximately $2.4 \times 10^{5}$ 
hPDL cells were suspended within fibrinogen solution and pipetted into one scaffold, then incubated for $6 \mathrm{~h}$ before surgery. Thrombin solution was then added at a thrombin/ fibrinogen volume ratio of 1:5 during surgery.

After rinsing twice with PBS, fiber-guiding scaffolds were stored in $70 \%$ ethanol, placed into cell culture media, and incubated at $37^{\circ} \mathrm{C}$. Each region of the scaffold contained the following amount of cells: The PDL interface $(2 \mu \mathrm{L})$ and the bone region $(4 \mu \mathrm{L})$ housed $0.4 \times 10^{5} \mathrm{hPDL}$ cells and $0.6 \times 10^{5}$ hPDL cells, respectively.

\section{Animal surgery and scaffold implantation}

Athymic nude rats $(n=48)$ weighing $\sim 225-250 \mathrm{~g}$ (Charles River Laboratories International, Inc., Strain code: 316) were anesthetized with ketamine $(90 \mathrm{mg} / \mathrm{kg}$; Fort Dodge Animal Health) and xylazine $(10 \mathrm{mg} / \mathrm{kg}$; Lloyd, Inc.). Standardized periodontal osseous fenestration defects were surgically created, and the fiber-guiding scaffold structure with cellsuspended fibrin gel matrix was placed into the periodontal defect site as previously described. ${ }^{36}$ Analgesic ( $5 \mathrm{mg} / \mathrm{kg}$; Rimadyl ${ }^{\circledR}$ [Carprofen], Pfizer Animal Health,) was administered subcutaneously for $24 \mathrm{~h}$ postsurgery, and ampicillin antibiotic water $(0.268 \mathrm{mg} / \mathrm{L})$ was provided once a day for $\sim 10$ days postsurgery. Animals were checked for signs of infection every 2-3 days, and surgical staples were removed on the 14th day.

\section{Quantification of mineralized tissue formation}

Animals were euthanized at 3 and 6 week time points for the harvesting of mandibular specimens. Harvested specimens were fixed in $10 \%$ buffered formalin phosphate solu- tion (Fisher Scientific) for approximately 2 days, then stored in $70 \%$ ethanol at $4{ }^{\circ} \mathrm{C}$. These specimens were scanned in PBS with $18 \times 18 \times 18 \mu \mathrm{m}^{3}$ voxel size at previously mentioned conditions. Once scanning of the specimens was complete, MicroView Analysis +2.1.2 software was utilized to create region-of-interest (ROI) contours around the defect site to quantify regeneration of dental-supporting structures. ${ }^{37}$ Anatomical landmarks used for drawing the ROI borders were created as previously described, and a 3-D construct was generated by interpolating drawn contours. ${ }^{36}$ Bone volume fraction (BVF) and tissue mineral density (TMD) were used as parameters for quantifying regenerated bone quantity and quality.

\section{Histological evaluation and morphological analysis of regenerated tissue interface}

For second harmonic generation (SHG) of collagen type-I, transverse thin sections ( $<1 \mathrm{~mm}$ thick) were cut off between the cementoenamel junction and root apex to include both soft and hard tissues (Fig. 3). Emission-detection settings (software gain, image resolution) were standardized along with the scanning depth and step size $(\approx 100 \mu \mathrm{m}$ at $\approx 1.4 \mu \mathrm{m})$. A field emission scanning electron microscope (FESEM; AMRAY 1910, Amray, Inc.) was utilized for morphological analysis of the PDL/cementum tissue interface (Fig. 4). After SHG imaging, specimens were dried, mounted on a specimen stub, and sputter coated with gold/palladium alloy. Scanning electron microscopic (SEM) imaging proceeded in the area of interest at $5.00 \mathrm{kV}$. For histological evaluation, the harvested mandibulae were decalcified in $10 \%$ ethylene diaminetetraacetic acid
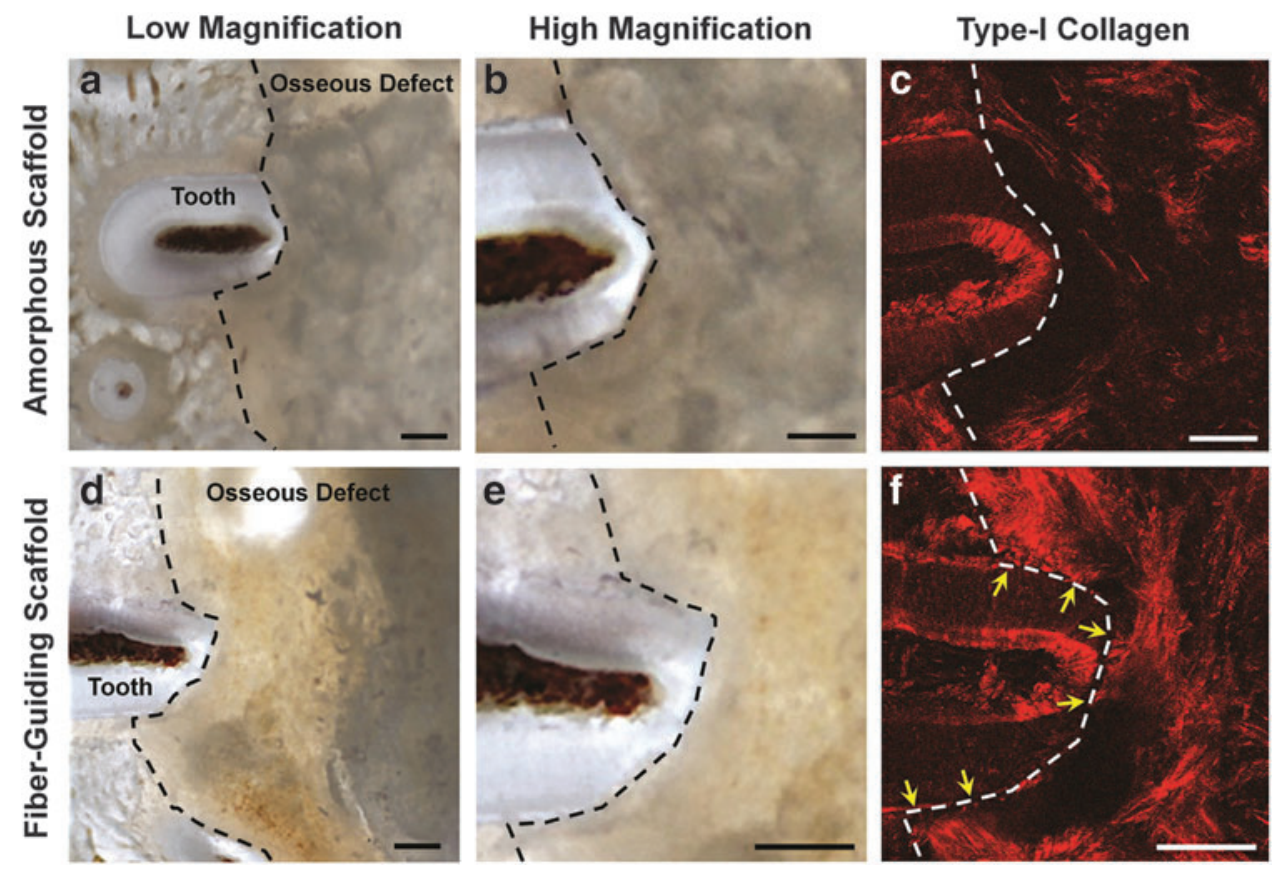

FIG. 3. Generation of dental-ligament complexes as evidenced by type-I collagen fluorescence image analysis. The freshly sectioned images of each scaffold group $(\mathbf{a}, \mathbf{b}, \mathbf{d}, \mathbf{e})$ had the limitation to analyze fibrous connective tissue formation and orientation, so type-I collagen fluorescence $(\mathbf{c}, \mathbf{f})$ was utilized to detect fiber generation in the PDL interface. (c) Amorphous, random-porous scaffold demonstrated no fiber formation and type-I collagen expression around the PDL interface. However, (f) yellow arrows that indicated type-I collagenous fiber bundles were expressed with the anchorage to the tooth dentin surface and oriented perpendicularly or obliquely. Black-dashed lines represented borders of the osseous fenestration defects. Scale bars: $50 \mu \mathrm{m}$. Color images available online at www.liebertpub.com/tec 
FIG. 4. SEM images. The amorphous, random-porous scaffold $\mathbf{( a ,} \mathbf{b})$ displays limited-no connective or fibrous tissue within the PDL interface, while the fiberguiding scaffold (c, d) showed that fibrous tissues were densely generated in the PDL region and osseous defect site. Moreover, (d-i) a white dashed arrow represented that the fibrous bundle was oriented along the PDL-guiding architecture of the fiber-guiding scaffold with a connection on the dentin surface (yellow arrows). (d-ii) Yellow arrows indicate collagen-like fibers and their anchorages on the tooth dentin surfaces. Solid yellow lines and triangles were the borders of cementum-removed dentin surface, red-dashed lines were PDL architectures, and the blue-dashed border was the bone region of the fiberguiding scaffold. Color images available online at www.liebertpub.com/tec
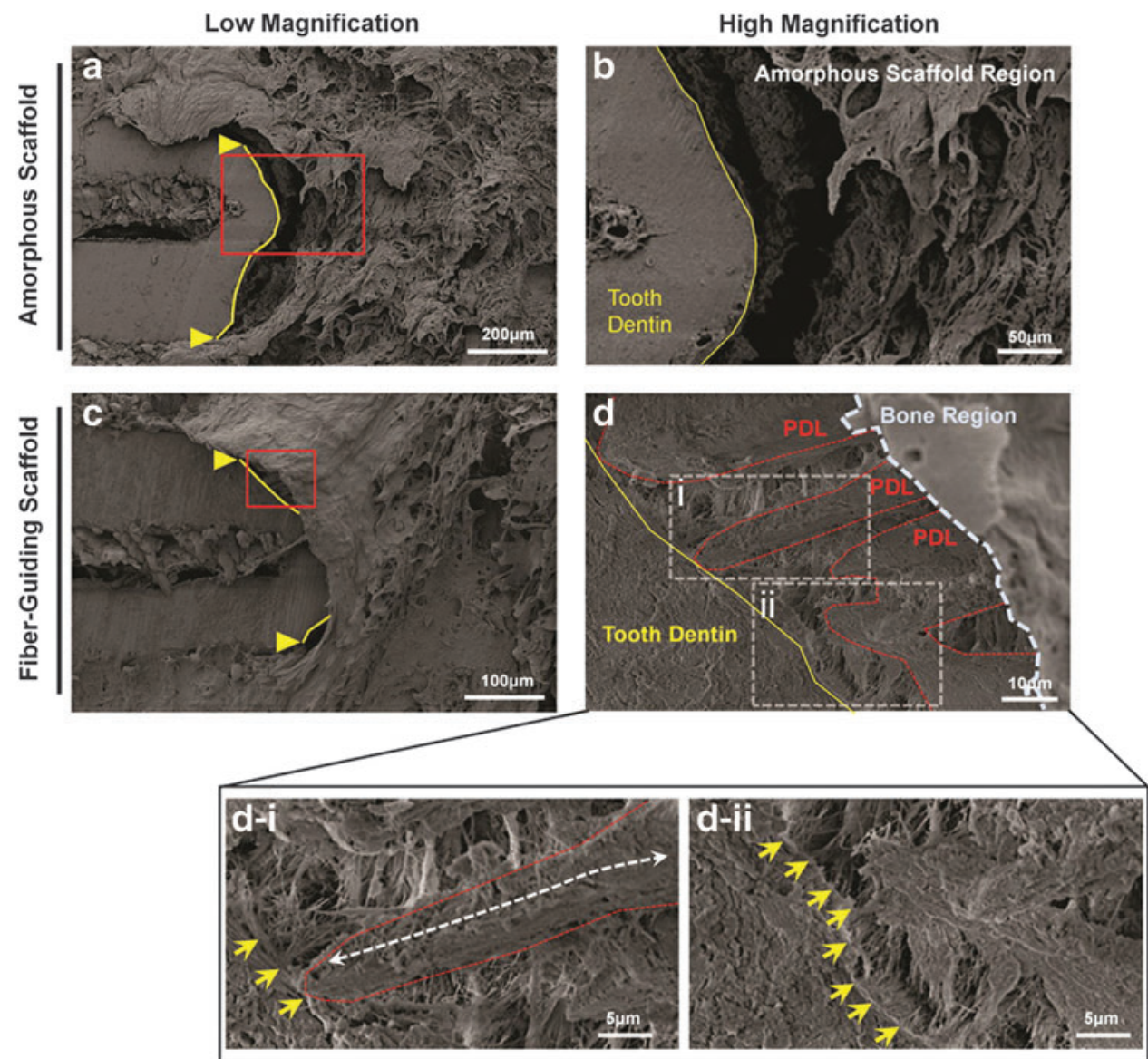

(EDTA; Sigma-Aldrich) for 4 weeks and embedded in paraffin blocks for histological sectioning in the coronal plane (Fig. 5). After hematoxylin and eosin (H\&E) staining, ImageJ software $(\mathrm{NIH})$ was used to measure angular orientation of fibrous ligament tissues.

\section{Statistical analysis}

PASW Statistics 17.0 (Software for statistics; SPSS, Inc., www.spss.com) was utilized for a statistical comparison among all experimental groups. All of the results were
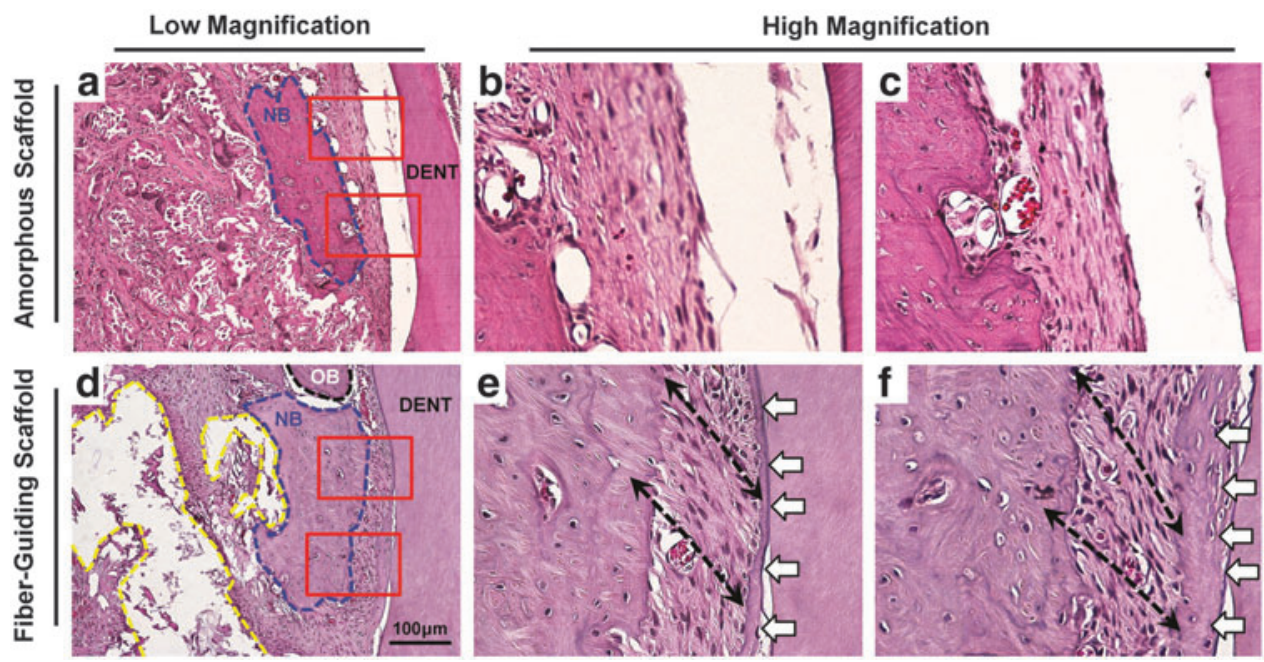

FIG. 5. Histological analysis of neogenic tissue morphologies and fibrous tissue organization with amorphous and fiberguiding scaffolds. The randomly organized amorphous scaffold $(\mathrm{a}-\mathrm{c})$ displayed newly formed bone tissues (blue-dashed contour; NB) and densely packed fibrous tissues between dentin surface and new bone tissue. However, fibrous tissue structures had disorganized orientation to the root surface as compared with mature ligament structure and no tissue attachment on root surface. The fiber-guiding scaffold (d-f) showed new bone formation (blue-dashed contour), specific oriented fibrous bundle formation (black-dashed arrow in $\mathbf{e}, \mathbf{f}$ ), and mineralized tissue deposition, which white arrows indicate on the root surface. Color images available online at www.liebertpub.com/tec 
significantly different with the $\alpha$-value set at 0.05 level of significance.

\section{Results}

\section{Adaptation ratio using micro-CT}

For the scaffold-defect adaptability analysis, three randomly selected $35 \% \mathrm{BaSO}_{4}$-stained scaffolds from each wax mold were scanned using micro $\mathrm{CT}$, evaluated, and $\mathrm{d}^{\text {gap }}$ and $\mathrm{d}^{\mathrm{PDL}}$ were used to calculate the adaptation ratio-data not shown. ${ }^{36}$ The average scaffold-root surface adaptation ratio was $0.924 \pm 0.031$ (mean \pm standard deviation). No statistical differences were found between scaffolds from three different wax molds using an ANOVA test $(p=0.3)$. Moreover, Cronbach's Alpha Coefficient ( $\alpha$-value) was utilized to evaluate manufacturing reliability and internal consistency of polymeric scaffolds. Statistically relevant reliability and consistency of the scaffold adaptation ratios $(\alpha$-value $=0.738)$ indicated that rapid-prototyped, customized fiber-guiding scaffolds had a significantly high defect conformation.

\section{Bone parameter analyses using micro CT}

The ROI was created to assess the quantification of mineralized tooth-supporting tissue formation within standardized surgical defects at 3- and 6 weeks postsurgery. On analysis of the 3-D ROI, mineralized tissues in fiber-guiding scaffolds were regenerated with significant differences between 3- and 6-week time points $\left(p^{\mathrm{BVF}}=0.009, p^{\mathrm{TMD}}=0.040\right){ }^{36}$

\section{Type-I collagen formation and orientation} to the root surface within periodontal defect

Multiphoton microscopic imaging of collagen based on SHG microscopy enables imaging of the collagen molecular organization. ${ }^{41}$ Collagen has a highly crystalline triple-helix structure that makes it exceptionally efficient for this technique, ${ }^{42}$ specifically for collagen type-I detection. ${ }^{43}$
SHG images were used to qualitatively analyze for type-I collagen formation and orientation. Type-I collagen is one of the main elements in the Sharpey's fiber bundle that is anchored to the mineralized tissue surfaces, tooth root surface, and alveolar bone surface. In Figure $3 \mathrm{f}$, there were three bundle-like structures, which were regenerated following the PDL topologies of the fiber-guiding scaffold. Yellow arrows indicate that three different type-I collagen bundles were connected, perpendicularly oriented to the root surface, and extended into the bone region. However, the amorphous scaffold had no collagenous structures around the tooth root surface and PDL interface. Therefore, customized PDL geometries in the fiber-guiding scaffold construct guide cell orientation, as well as type-I collagen fibrous bundle formation and alignment. ${ }^{36}$

\section{SEM qualification analysis for collagenous fiber formation and orientation}

Regenerated PDL morphologies by amorphous and fiberguiding scaffolds were examined using FESEM. Minimum fibrous connective tissue was formed between the tooth root surface and the bone region of a scaffold in the amorphous scaffold design (Fig. 4a, b). However, the fiber-guiding scaffold showed ligament-like tissue formation and alignment following designed PDL geometries (Fig. 4c, d). This morphological analysis demonstrated that geometrical design and its approach influenced a different organization of regenerated tissue and controlled the orientation/directionality of fibrous bundles.

\section{Histological analysis for multiple tissue formation and orchestration around periodontal defect sites}

H\&E staining was used to histomorphometrically analyze regenerated tissues within periodontal defect sites. Transversely sectioned images demonstrated that multiple tissues, PDL, and newly formed mineralized tissues were

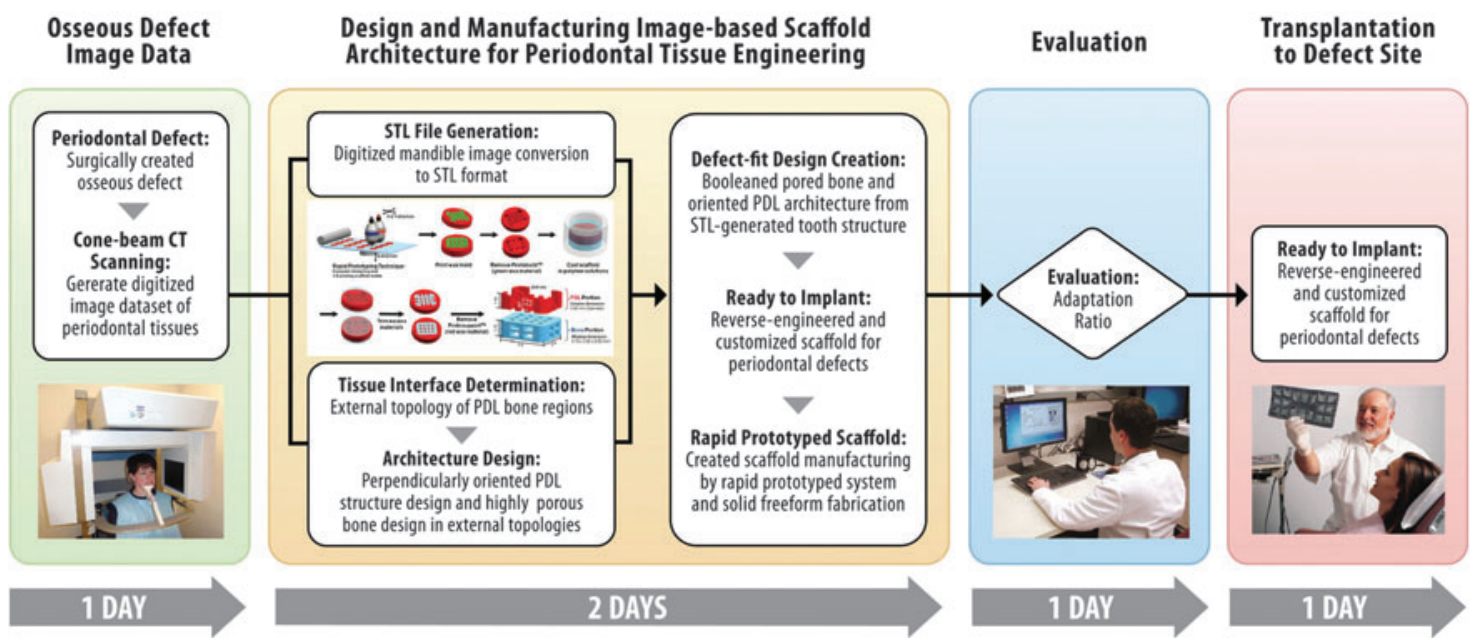

FIG. 6. Flow chart for the fiber-guiding scaffold design, manufacture, and potential application in the future A cone-beam CT scan is taken of the defect site for digitized image dataset. Based on image data, computed design aided is utilized to design the defect-fit, fiber-guiding scaffolds with specific oriented PDL geometries, and wax molds are manufactured by the 3-D printer. Poly- $\varepsilon$-caprolactone was cast in molds, and three scaffolds per mold were chosen to evaluate the adaptation ratio. If the ratio is $>90 \%$, this rapid prototyping technique has the potential to be utilized in future scaffold manufacturing applications for micron-level, multiple-tissue complex regeneration (periodontal complexes). Color images available online at www.liebertpub.com/tec 
regenerated around the tooth root defect (Fig. 5). In the amorphous scaffold group, fibrous tissue was regenerated but with parallel orientation and had no attachment to the cementum-removed root surface (Fig. 5a-c). In contrast, PDL architectures in the fiber-guiding scaffold construct guided fiber alignment and oblique orientation to the root surface (Fig. 5d-f). Moreover, in the interfacial region, the PDL-tooth root, and PDL-regenerated bone, fibrous bundles were attached and anchored similar to mature, healthy periodontal complexes (Fig. 5e, f). We analyzed 12 fiber-guiding scaffolds and 12 amorphous scaffolds, per time point. All fiber-guiding scaffolds at 3 weeks $(n=12)$ and 9 out of the 12 fiber-guiding scaffolds at 6 weeks had PDL architectures. Consequently, designed PDL architectures in fiber-guiding scaffolds promoted proper fiber regeneration and orientation with periodontal tissue integration under biomechanical loading conditions.

\section{Discussion}

Tissue engineering paradigms have revolutionized a shift from prosthetics to tissue regeneration with biomaterials. ${ }^{1,2}$ However, it remains difficult to achieve multi-tissue regeneration and integration in musculoskeletal tissue regeneration. There are limited studies for multiple tissue regeneration and interfacial tissue integration for functional constructs of neogenic complexes such as ligament bone or cartilage bone. ${ }^{5,6,25,44}$ In recent studies, multi-phasic scaffolds or physical gradient constructs have been investigated as vehicles for facilitating multiple tissue formation within a single system, ${ }^{7,8}$ as well as bioactive molecule-induced tissue morphogenesis. ${ }^{9}$ Various in vitro approaches emphasize the strong potential of geometric control for tissue regeneration and morphogenesis with diverse types of cells. ${ }^{10-12}$ A limited number of research studies report on the geometric influences on in vivo spatiotemporal tissue regeneration and orchestration, and, more specifically, functional recovery due to unpredictable and uncontrollable reorganization and rearrangement of regenerated tissues. ${ }^{13,44}$

One of the most important requirements for a functional periodontal complex is the alignment of type-I collagen bundles/PDL fibers perpendicular to the tooth root surface. We previously developed an image-based, fiber-guiding scaffolding system, ${ }^{36,37}$ which facilitated the formation of ligamentous tissue with a perpendicular/oblique orientation, compartmentalized bone, and ligament regeneration with functional tissue integration. Furthermore, we used the scaffold topology to control tissue morphology and fibrous tissue formation using hPDL cells under biomechanical, masticatory environments. Conventional, amorphous salt-leached scaffolds were used as controls for fiber-guiding scaffolds.

The principal finding in this study was the geometric control of tissue formation in the periodontal in vivo system within a physiologically complex environment. In particular, the PDL architecture of the fiber-guiding construct promoted both cell and tissue organization, as well as the formation of type-I collagen bundles, also known as Sharpey's fibers (Fig. $3 e$ ), oriented perpendicular to the tooth root surface (Fig. 4d). Periodontal Sharpey's fibers are continuous collagen fibers within the PDL embedded into the cementum and alveolar bone that serve as anchors between mineralized tissues. In addition, Sharpey's fibers respond to mechanical forces to control tooth movement and also play a vital role in the functional maintenance of alveolar bone. Type-I collagen staining demonstrated the formation of three separate collagen fibrous bundles (Fig. 3), corresponding to the three PDL architectures of the fiber-guiding scaffold. Interestingly, type-I collagen fluorescence staining (Fig. 3f) and SEM imaging (Fig. 4c, d) showed newly formed fibrous bundles embedded within the surface tooth dentin. Therefore, the fiber-guiding scaffold served as a guiding platform for the formation of collagen matrices with proper anatomy and orientation. Looking at regenerated collagen structures, fibrous bundles were anchored into a cementum-like mineralized layer on the root surface (Fig. 4d) that closely resembled functional periodontal structures.

Anatomically designed scaffolds with biomimetic geometries facilitated the regeneration, arrangement, and organization of multiple periodontal tissues. Most notably, the scaffold architecture directed the integration of PDL and mineralized tissue structures, resulting in functional tissue interfaces that mimic natural anatomy. The physical and geometric approaches behind this principal methodology can be applied toward achieving tissue interface regeneration. Furthermore, novel designs in surface geometry and topology provide a wide spectrum of applications toward multiple tissue regeneration in situations with nonoptimal bioactive chemical stimulation to be investigated in future preclinical and clinical studies (Fig. 6).

\section{Acknowledgments}

The work contained in this article was supported by NIH/ NIDCR DE 13397-11. The authors acknowledge the assistance received from Chris Jung with regard to the preparation of the figures (University of Michigan). They also thank Erika Benavides for providing clinical images used in article figures.

\section{Disclosure Statement}

The authors declare no competing financial interests.

\section{References}

1. Huebsch, N., and Mooney, D.J. Inspiration and application in the evolution of biomaterials. Nature 462, 426, 2009.

2. Ratner, B.D., and Bryant, S.J. Biomaterials: where we have been and where we are going. Annu Rev Biomed Eng 6, 41, 2004.

3. Lee, C.H., Cook, J.L., Mendelson, A., Moioli, E.K., Yao, H., and Mao, J.J. Regeneration of the articular surface of the rabbit synovial joint by cell homing: a proof of concept study. Lancet 376, 440, 2010.

4. Lu, H.H., Subramony, S.D., Boushell, M.K., and Zhang, X. Tissue engineering strategies for the regeneration of orthopedic interfaces. Ann Biomed Eng 38, 2142, 2010.

5. Yang, P.J., and Temenoff, J.S. Engineering orthopedic tissue interfaces. Tissue Eng Part B Rev 15, 127, 2009.

6. Moffat, K.L., Sun, W.H., Pena, P.E., Chahine, N.O., Doty, S.B., Ateshian, G.A., et al. Characterization of the structurefunction relationship at the ligament-to-bone interface. Proc Natl Acad Sci U S A 105, 7947, 2008.

7. Phillips, J.E., Burns, K.L., Le Doux, J.M., Guldberg, R.E., and Garcia, A.J. Engineering graded tissue interfaces. Proc Natl Acad Sci U S A 105, 12170, 2008. 
8. Schek, R.M., Taboas, J.M., Segvich, S.J., Hollister, S.J., and Krebsbach, P.H. Engineered osteochondral grafts using biphasic composite solid free-form fabricated scaffolds. Tissue Eng 10, 1376, 2004.

9. Koh, J.T., Zhao, Z., Wang, Z., Lewis, I.S., Krebsbach, P.H., and Franceschi, R.T. Combinatorial gene therapy with BMP2/7 enhances cranial bone regeneration. J Dent Res 87, 845, 2008.

10. Raghavan, S., Nelson, C.M., Baranski, J.D., Lim, E., and Chen, C.S. Geometrically controlled endothelial tubulogenesis in micropatterned gels. Tissue Eng Part A 16, 2255, 2010.

11. Grayson, W.L., Frohlich, M., Yeager, K., Bhumiratana, S., Chan, M.E., Cannizzaro, C., et al. Engineering anatomically shaped human bone grafts. Proc Natl Acad Sci U S A 107, 3299, 2010.

12. Rumpler, M., Woesz, A., Dunlop, J.W., van Dongen, J.T., and Fratzl, P. The effect of geometry on three-dimensional tissue growth. J R Soc Interface 5, 1173, 2008.

13. Mao, J.J., Giannobile, W.V., Helms, J.A., Hollister, S.J., Krebsbach, P.H., Longaker, M.T., et al. Craniofacial tissue engineering by stem cells. J Dent Res 85, 966, 2006.

14. Rios, H.F., Ma, D., Xie, Y., Giannobile, W.V., Bonewald, L.F., Conway, S.J., et al. Periostin is essential for the integrity and function of the periodontal ligament during occlusal loading in mice. J Periodontol 79, 1480, 2008.

15. Flores, M.G., Yashiro, R., Washio, K., Yamato, M., Okano, T., and Ishikawa, I. Periodontal ligament cell sheet promotes periodontal regeneration in athymic rats. J Clin Periodontol 35, 1066, 2008.

16. Nagatomo, K., Komaki, M., Sekiya, I., Sakaguchi, Y., Noguchi, K., Oda, S., et al. Stem cell properties of human periodontal ligament cells. J Periodontal Res 41, 303, 2006.

17. Seo, B.M., Miura, M., Gronthos, S., Bartold, P.M., Batouli, S., Brahim, J., et al. Investigation of multipotent postnatal stem cells from human periodontal ligament. Lancet 364, 149, 2004.

18. Shimono, M., Ishikawa, T., Ishikawa, H., Matsuzaki, H., Hashimoto, S., Muramatsu, T., et al. Regulatory mechanisms of periodontal regeneration. Microsc Res Tech 60, 491, 2003.

19. Oshima, M., Mizuno, M., Imamura, A., Ogawa, M., Yasukawa, M., Yamazaki, H., et al. Functional tooth regeneration using a bioengineered tooth unit as a mature organ replacement regenerative therapy. PLoS One 6, e21531. 2011.

20. Chen, R., Chiba, M., Mori, S., Fukumoto, M., and Kodama, T. Periodontal gene transfer by ultrasound and nano/ microbubbles. J Dent Res 88, 1008, 2009.

21. Taba, M., Jr., Jin, Q., Sugai, J.V., and Giannobile, W.V. Current concepts in periodontal bioengineering. Orthod Craniofac Res 8, 292, 2005.

22. Jin, Q.M., Anusaksathien, O., Webb, S.A., Rutherford, R.B., and Giannobile, W.V. Gene therapy of bone morphogenetic protein for periodontal tissue engineering. J Periodontol 74, 202, 2003.

23. Haze, A., Taylor, A.L., Haegewald, S., Leiser, Y., Shay, B., Rosenfeld, E., et al. Regeneration of bone and periodontal ligament induced by recombinant amelogenin after periodontitis. J Cell Mol Med 13, 1110, 2009.

24. Hong, L., and Mao, J.J. Tissue-engineered rabbit cranial suture from autologous fibroblasts and BMP2. J Dent Res 83, 751, 2004.

25. Akman, A.C., Tigli, R.S., Gumusderelioglu, M., and Nohutcu, R.M. bFGF-loaded HA-chitosan: a promising scaffold for periodontal tissue engineering. J Biomed Mater Res A 92, 953, 2010.
26. Iwata, T., Yamato, M., Tsuchioka, H., Takagi, R., Mukobata, S., Washio, K., et al. Periodontal regeneration with multilayered periodontal ligament-derived cell sheets in a canine model. Biomaterials 30, 2716, 2009.

27. Spalazzi, J.P., Dagher, E., Doty, S.B., Guo, X.E., Rodeo, S.A., and $\mathrm{Lu}, \mathrm{H} . \mathrm{H}$. In vivo evaluation of a multiphased scaffold designed for orthopaedic interface tissue engineering and soft tissue-to-bone integration. J Biomed Mater Res A 86, 1, 2008.

28. Taboas, J.M., Maddox, R.D., Krebsbach, P.H., and Hollister, S.J. Indirect solid free form fabrication of local and global porous, biomimetic and composite 3D polymer-ceramic scaffolds. Biomaterials 24, 181, 2003.

29. Um, Y.J., Jung, U.W., Chae, G.J., Kim, C.S., Lee, Y.K., Cho, K.S., et al. The effects of hydroxyapatite/calcium phosphate glass scaffold and its surface modification with bovine serum albumin on 1-wall intrabony defects of beagle dogs: a preliminary study. Biomed Mater 3, 044113, 2008.

30. Shi, H., Ma, J., Zhao, N., Chen, Y., and Liao, Y. Periodontal regeneration in experimentally-induced alveolar bone dehiscence by an improved porous biphasic calcium phosphate ceramic in beagle dogs. J Mater Sci Mater Med 19, 3515, 2008.

31. Kaigler, D., Cirelli, J.A., and Giannobile, W.V. Growth factor delivery for oral and periodontal tissue engineering. Expert Opin Drug Deliv 3, 647, 2006.

32. Murakami, S., Takayama, S., Ikezawa, K., Shimabukuro, Y., Kitamura, M., Nozaki, T., et al. Regeneration of periodontal tissues by basic fibroblast growth factor. J Periodontal Res 34, 425, 1999.

33. Vaquette, C., Fan, W., Xiao, Y., Hamlet, S., Hutmacher, D.W., and Ivanovski, S. A biphasic scaffold design combined with cell sheet technology for simultaneous regeneration of alveolar bone/periodontal ligament complex. Biomaterials 33, 5560, 2012.

34. Pellegrini, G., Seol, Y.J., Gruber, R., and Giannobile, W.V. Pre-clinical models for oral and periodontal reconstructive therapies. J Dent Res 88, 1065, 2009.

35. Oortgiesen, D.A., Meijer, G.J., Bronckers, A.L., Walboomers, X.F., and Jansen, J.A. Fenestration defects in the rabbit jaw: an inadequate model for studying periodontal regeneration. Tissue Eng Part C Methods 16, 133, 2010.

36. Park, C.H., Rios, H.F., Jin, Q., Sugai, J.V., Padial-Molina, M., Taut, A.D., et al. Tissue engineering bone-ligament complexes using fiber-guiding scaffolds. Biomaterials 33, 137, 2012.

37. Park, C.H., Rios, H.F., Jin, Q., Bland, M.E., Flanagan, C.L., Hollister, S.J., et al. Biomimetic hybrid scaffolds for engineering human tooth-ligament interfaces. Biomaterials 31, 5945, 2010.

38. Padial-Molina, M., Marchesan, J.T., Taut, A.D., Jin, Q., Giannobile, W.V., and Rios, H.F. Methods to validate toothsupporting regenerative therapies. Methods Mol Biol 887, 135, 2012.

39. Kallai, I., Mizrahi, O., Tawackoli, W., Gazit, Z., Pelled, G., and Gazit, D. Microcomputed tomography-based structural analysis of various bone tissue regeneration models. Nat Protoc 6, 105, 2011.

40. Rios, H.F., and Giannobile, W.V. Chapter 7: preclinical protocols for periodontal regeneration. In: Giannobile, W.V., and Nevins, M., eds. Osteology Guidelines for Oral and Maxillofacial Regeneration. Chicago: Quintessence Publishing, 2011, pp. 77-101.

41. Zipfel, W.R., Williams, R.M., Christie, R., Nikitin, A.Y., Hyman, B.T., and Webb, W.W. Live tissue intrinsic emission microscopy using multiphoton-excited native fluorescence 
and second harmonic generation. Proc Natl Acad Sci U S A 100, 7075, 2003.

42. Jiang, X., Zhong, J., Liu, Y., Yu, H., Zhuo, S., and Chen, J. Two-photon fluorescence and second-harmonic generation imaging of collagen in human tissue based on multiphoton microscopy. Scanning 33, 53, 2011.

43. Georgakoudi, I., Jacobson, B.C., Muller, M.G., Sheets, E.E., Badizadegan, K., Carr-Locke, D.L., et al. NAD $(\mathrm{P}) \mathrm{H}$ and collagen as in vivo quantitative fluorescent biomarkers of epithelial precancerous changes. Cancer Res 62, 682, 2002.

44. Ho, S.P., Kurylo, M.P., Fong, T.K., Lee, S.S., Wagner, H.D., Ryder, M.I., et al. The biomechanical characteristics of the bone-periodontal ligament-cementum complex. Biomaterials 31, 6635, 2010.
Address correspondence to: William V. Giannobile, DDS, DMedSc Department of Periodontics and Oral Medicine School of Dentistry University of Michigan 1011 N. University Ave. Ann Arbor, MI 48109-1078

E-mail: wgiannob@umich.edu

Received: October 2, 2013

Accepted: October 22, 2013

Online Publication Date: December 13, 2013 